\title{
The influence of Kanthal wire surface defects on the formation of Si nanolayer deposited by PVD method
}

\author{
MieCZySŁAW SZCZYPIŃski ${ }^{1}$, KAZIMIERZ RESZKA ${ }^{2}$, MichaŁ M. SZCZYPIŃSKi ${ }^{3, *}$ \\ ${ }^{1}$ PPHU TERMEX sp. z o.o., 75-213 Koszalin, Poland \\ ${ }^{2}$ Koszalin University of Technology, 75-453 Koszalin, Poland \\ ${ }^{3}$ Technical University of Liberec, 46117 Liberec, Czech Republic
}

\begin{abstract}
The subject of this research is the structure of a Si nanolayer deposited on a FeCrAl wire surface by means of magnetron sputtering method. Si layer was selected as one of possible protections of the wire surface against excessive corrosive-erosive wear. In order to increase the power necessary for the DC discharge of the magnetron with Si cathode, a second magnetron with an aluminum disc as a cathode was used. The wire was attached to a carousel holder to ensure its rotation around the magnetron. The thickness of the deposited layers was about $150 \mathrm{~nm}$. A wire surface examination indicated the presence of defects such as gaps between grains, cavities as well as severely deformed grains of surface layer. The research was conducted on the sample sections which had been prepared by focused ion beam method (FIB). The technique of transmission microscopy, which was used for observation, allowed us to obtain images in bright field (BF), dark field (DF), as well as in high resolution (HREM). The studies were also performed on the wire surface after the cutting process of the expanded polystyrene blocks. A metallographic optical microscope Nikon MA200 with a large depth of field was used for the examination which showed the presence of carbon deposit products. Additionally, a composition microanalysis was carried out along the line within selected areas of samples, with the use of energy dispersive spectroscopy (EDS). A large impact of wire surface defects on Si layer forming was found as well as a high direct homogeneous growth. The examination of the sections indicated the existence of a mechanism of defects sealed by Si layer, where directionality of grains growth in these areas revealed the tendency for vertical location relative to defects surface. Consequently, closed nanopores, i.e. spaces not covered with Si layer, were created. It is a characteristic feature of areas with defects covered with an oxide film created in a natural way.
\end{abstract}

Keywords: PVD; nanolayers; microwire; corrosion; EPS

\section{Introduction}

In addition to conventional use in heating sector, resistive wires are used on industrial-scale in the process of forming panels of expanded polystyrene, i.e. EPS (commonly known as Styrofoam). The wires are stretched on a special frame in the form of strings, in the distances determined by the thickness of plates, and after being warmed to a temperature of ca. $160{ }^{\circ} \mathrm{C}$, they are moved to a fixed block or the block is moved to a fixed frame, depending on the variant applied. Due to wires thermal linear expansion, they are tensioned with a force from $8 \mathrm{~N}$ to $25 \mathrm{~N}$, depending on their length. Additionally, there are the stresses related to the resistance of melting material, caused by

*E-mail: michal.szczypinski@tul.cz the viscosity of polystyrene in the narrow melting zone, the size of which depends on temperature of a wire (material coating a wire), and the width of a heat transfer zone (the distance to notmolten walls). Due to the extremely small diameter of applied wires, which is from $0.05 \mathrm{~mm}$ to $0.5 \mathrm{~mm}$, in comparison to their length from $800 \mathrm{~mm}$ to $1200 \mathrm{~mm}$, these changes are essentially of linear and surface nature, and concern volume to a lesser extent. Raising the temperature decreases the resistance force as the molten zone is extended, however, it results in the excessive production of liquid material, which, consequently, sticks the plates, when it is in the form of drops. A proper selection of the width of cutting zone is adjusted by the wire temperature and the speed of block movement, however, it does not eliminate the problem of the impact of melting polystyrene on the wire 
surface, and the nature and force of this impact determine the operating capabilities of a wire (wear time). Recent research has indicated that apart from temperature and kinematic conditions mentioned above, this impact is also influenced by homogeneity of molten material as well as the quality of wire surface.

In the industrial processing of EPS blocks, recycled granulate additive is commonly used, whose quality and homogeneity is not standardized due to the fact that it originates from various sources. Thus, various hardness, granulation and melting points of these materials make the selection and maintenance of constant parameters for cutting blocks difficult. Occasionally, it requires an increase of temperature and slowing the movement. For the conditions selected for a basic material, a granulate with a higher melting point increases the resistance to wire movement within the cutting zone. Moreover, in this zone, granules of recyclate, which were not fully molten in an appropriate time, are moving into the material and accumulate. In the extreme case it leads to cyclic deviations from the correct position of a wire, especially in the central zone, and, consequently, folding of panels surface during cutting. The increase in tensile stresses, friction and the presence of liquid and gaseous byproducts in the cutting zone, as well as the environment of the atmosphere enriched with the residues of steam coming from the blocks, which the wires contact with at the edges of the block cut, are the factors that determine erosive-corrosive processes. Disregarding the material properties, the impact of the factors mentioned above is strongly related to the quality of wire surface, roughness and density of defected structures of subsurface grains and pores.

\section{Experimental}

The research has been conducted on a SANDVIC Kanthal-D wire with a diameter of $0.2 \mathrm{~mm}$, which is used for cutting EPS blocks into suitable shapes. The share of the main components of an alloy, from which the wire was produced, was as follows:
4.8 wt.\% Al, 20.9 wt.\% Cr, 0.22 wt.\% Si, other additives $<1 \%$, and $\mathrm{Fe}$. On the basis of electron microscopy examination, it was found that the surface of the wire was covered by amorphous oxide nanolayer, which might have been formed naturally due to prolonged exposition to atmosphere and possibly during the technological process of the wire forming [1-3]. Examination of the wires surface as well as the profiles of breaking points has been performed with an optical metallographic microscope Nikon MA200 with a large depth of field. The inner wire structure and the sections of Si layer have been examined by means of scanning transmission electron microscope (TEM/STEM) TECNAI FEG Super TWIN $(200 \mathrm{kV})$ with the function of high resolution examination (HREM) and spectral analysis of EDX. Specimens in the form of thin films were prepared with FIB technique [4], using Quanta 3D device equipped with Omniprobe manipulator. In order to maintain the sharpness of the contour of the layers edges, the samples were sprayed with a carbon layer from electric arc in a vacuum before thinning.

In order to deposit the layers, Magnetin K01 vacuum device (Fig. 1) has been used, which is an original device developed by the authors. It is equipped with two planar-circular DC magnetrons located at right angle, wherein polycrystalline silicon and aluminum plates with a diameter of $55 \mathrm{~mm}$ are used as cathodes [5]. The distances between the substrate and the targets of $\mathrm{Al}$ and Si plates are $90 \mathrm{~mm}$ and $45 \mathrm{~mm}$, respectively. The generated power density in this system varies from $1.26 \mathrm{~W} / \mathrm{cm}^{2}$ to $2.10 \mathrm{~W} / \mathrm{cm}^{2}$ for $\mathrm{Al}$ cathode and from $10.1 \mathrm{~W} / \mathrm{cm}^{2}$ to $10.9 \mathrm{~W} / \mathrm{cm}^{2}$ for $\mathrm{Si}$ cathode. In case of $\mathrm{Si}$ sputtering, it was difficult to initiate the discharge in the magnetron and then to maintain its stable operation. The use of an additional Al discharge eliminated this problem. This solution enabled the realization of discharge support by Al cathode magnetron, working at low power $(0.03 \mathrm{~kW}$ to $0.05 \mathrm{~kW})$. The power of $\mathrm{Si}$ cathode magnetron was stabilized within $0.24 \mathrm{~kW}$ to $0.26 \mathrm{~kW}$ with an argon pressure of $\mathrm{p}_{(\mathrm{Ar})}=5 \times 10^{-1} \mathrm{~Pa}$. The wires were 
attached to a carousel holder, and the chamber was evacuated from atmosphere to pre-pressure of $\mathrm{p}_{\mathrm{w}}=4 \times 10^{-3} \mathrm{~Pa}$, and then it was filled with argon and the operational pressure was maintained at a constant level. Due to the use of deposited layers as protective layers on alloys containing aluminum (i.e. Kanthal D), the low Al content in the Si layers did not affect the protective properties of these layers, but it allowed a repeatable and stable initiation of the magnetron sputtering of the Si target, especially since it was on the same level as the content in the core alloy.

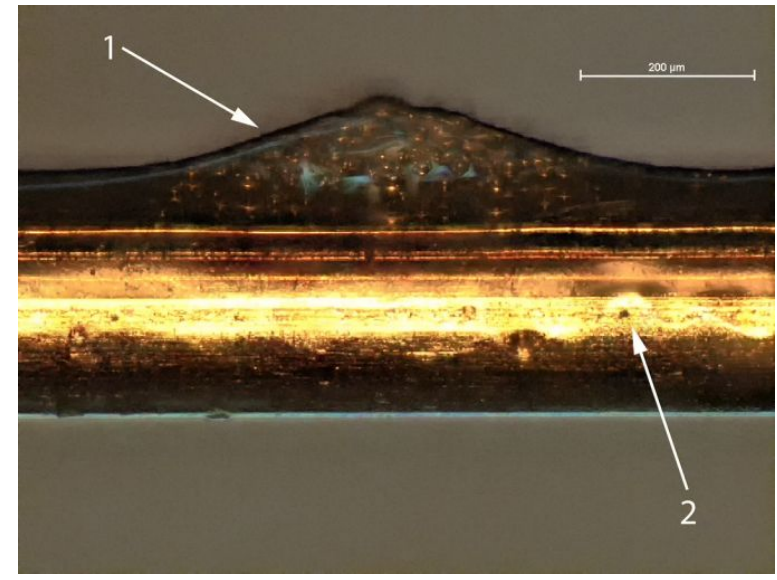

(a)

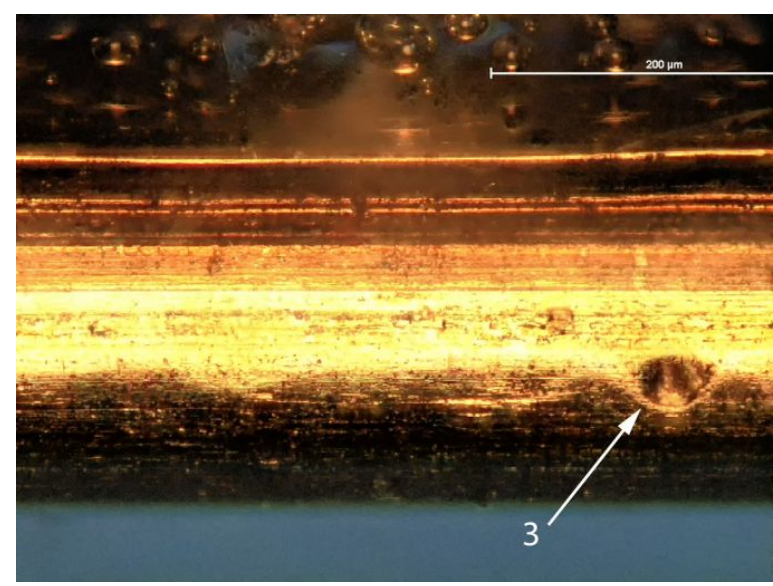

(b)

Fig. 2. Images obtained by optical metallographic microscope Nikon MA200; 1 - a residue of molten polystyrene, 2 - wide etching of a wire, 3 - etching pit.

K01 device system designed for deposition of $\mathrm{Si}$ nanolayers.

\section{Results and discussion}

Observation of the surface of longer sections of the wire (Fig. 2), which was broken during operation performed on the optical metallographic microscope, revealed the presence of a residue of molten polystyrene in the form of carbon deposit (1), wide etched space (2) as well as deep etching pits (3).

The ongoing process of uneven wear of the wire, which occurs from the surface, causes its rupture in the weakest points. The profile of a rupture point shown in Fig. 3, discloses a non-homogeneity of the inner structure. There is a considerable diversity of grains deformed by tension forces. The difference in height is over $50 \mu \mathrm{m}$.

The appearance of strongly etched points in the first row may be associated with numerous surface defects arising during the forming of the wire, (Fig. 4). The presence of loose grains (1), open pores from the surface (2), pores between grains (3), and oxides on the surface of the wire as well as in the cavities and between grains (4) in the surface layer, which may indicate the occurrence of corrosion between the grains, provides the conditions for faster corrosive-erosive wear of material under the movement of molten polystyrene on the wire. 


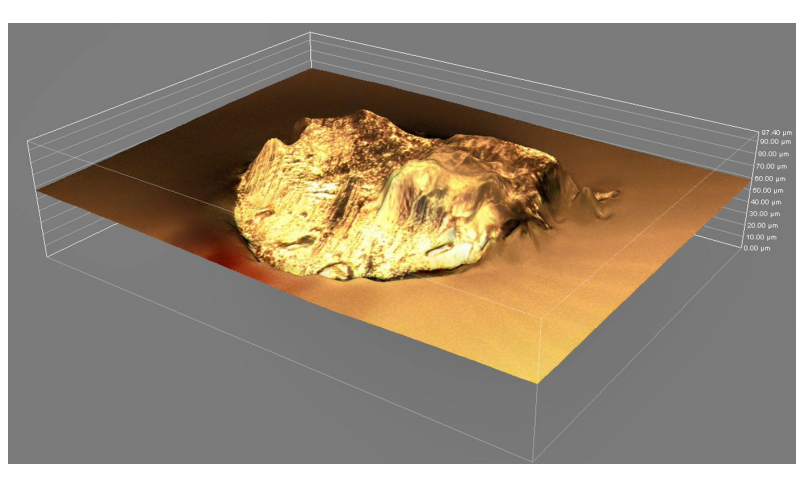

Fig. 3. Image obtained by optical metallographic microscope Nikon MA200; the profile of a rupture point with altitude scale.

Microanalysis along the line, performed during previous research has shown that the aluminum content in the deposited layer is similar to aluminum content in the alloy [6]. The examination of Si layer on the wire surface shows the differences in its structure, dependent on the area of deposition. The layer cross-sections in the areas free of surface defects (Fig. 5) indicate that they have grown in a uniform manner. Fine columnar grain boundaries (1), the zone adjacent to steel base composed of finer grains (2), and superstructured zone of columnar grains with developed bases and some expansion in the top (3) are visible. The structure of this system is likely influenced by amorphous natural oxide layer coating the wire, which enables the growth of silicon nanocrystals in the initial stage of deposition, and the formation of small grains on their basis. As the process proceeds, these grains coalesce and form more compact structures of a larger size.

The studies have shown that the grains tend to grow perpendicularly to the base (Fig. 6). In the convex areas, the grain bases are of small diameters and they expand into the zone of upper layer (2), while in the concave areas (3) the compensation grains are formed, which narrow in the upper zone. It may be assumed that as far as these areas are concerned, tensile stresses are present in the first case, and compression ones in the other. The frequency of the variation of stresses may contribute to the initiation of layer rupture. The arrangement of grains is also dependent on the surface corrugation, which results from

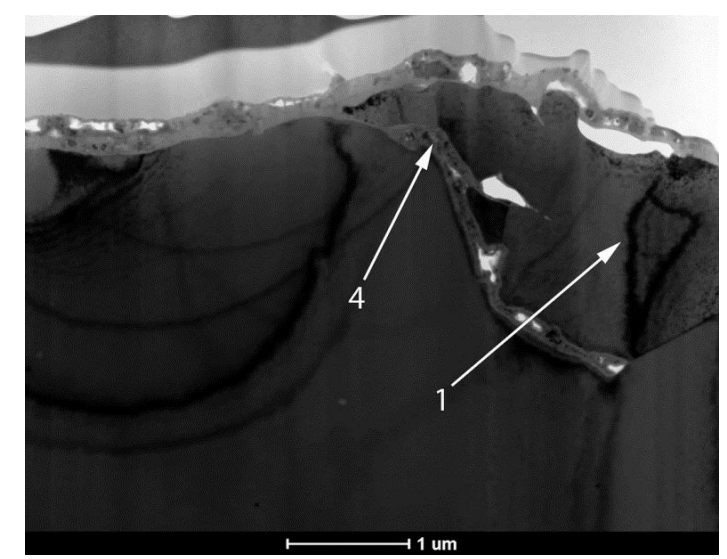

(a)

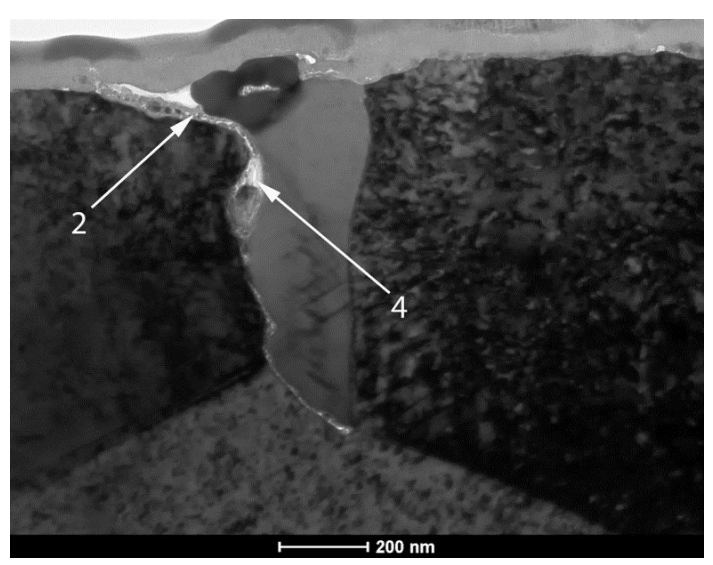

(b)

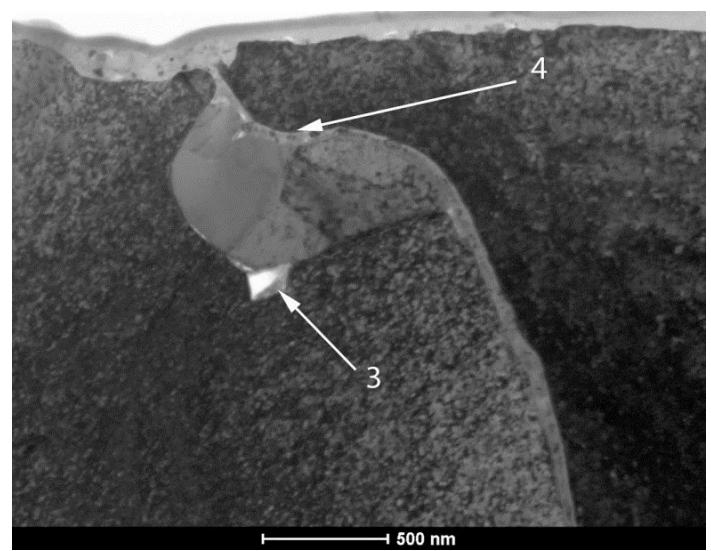

(c)

Fig. 4. TEM/STEM images of various forms of surface defects, arising during the forming of the wire.

the configuration of base grains, and additionally, the areas with a thicker oxide layer, occurring most commonly on grain boundaries (Fig. 7). The horizontal delamination of $\mathrm{Si}$ layer may 


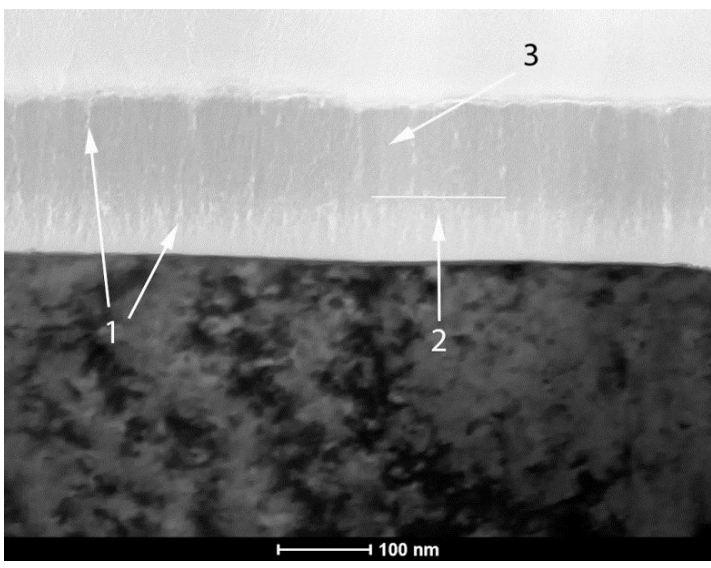

Fig. 5. TEM/STEM image of a fragment of Si layer section free of defects; 1 - boundaries between columnar grains, 2 - the zone of fine grains adjacent to the base, 3 - columnar grains.

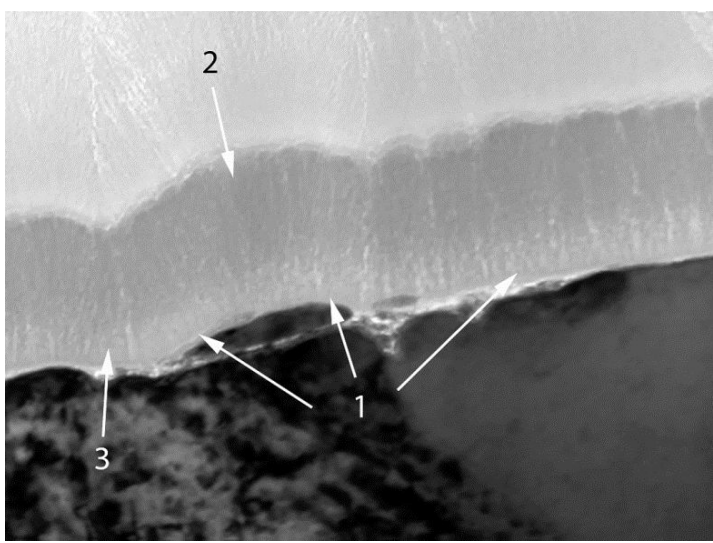

Fig. 6. TEM/STEM image of a fragment of Si layer section in a rolled section of the wire, 1 - directions of grain growth, 2 - the outline of a grain expanding upwardly, 3 - the outline of compensation grain.

occur over these areas (2), due to direct contact with the oxide layer and the layer created over the compensation grain.

It should be emphasized that both in the areas described above and in strongly defected areas with a large amount of oxides (Fig. 8), the deposited Si layer shows continuity. The unchanging nature of the grain growth oriented perpendicularly to the areas where crystallization was initiated, is maintained. It applies both to steel base (1), and to the space filled with a large amount

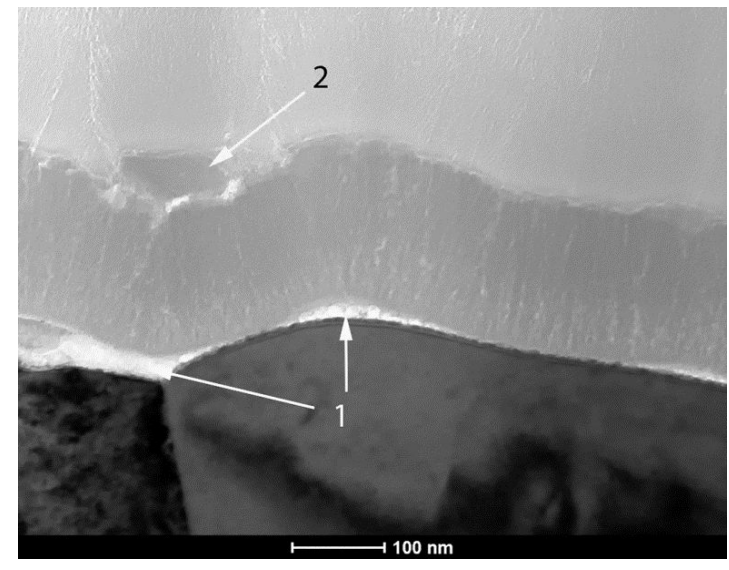

Fig. 7. TEM/STEM image of Si layer section of variable corrugation and level of oxide layer coating; 1 - areas of variate thickness of oxide layer, 2 - the fragment of delaminated Si layer.

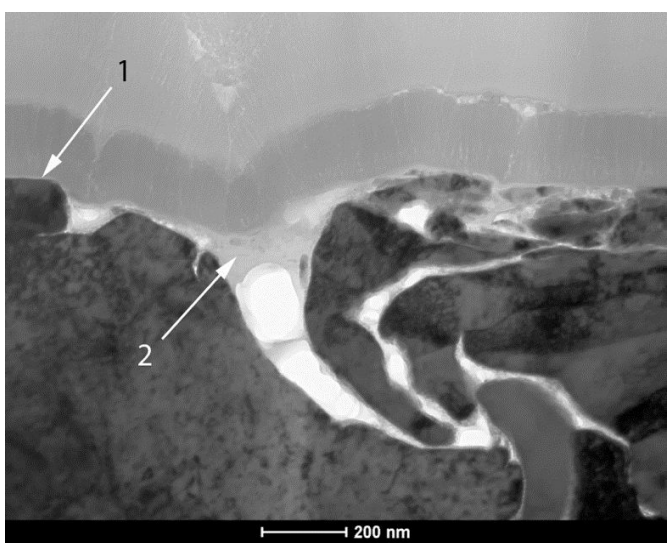

Fig. 8. TEM/STEM image of a Si layer section in the area of highly defected wire surface; 1 - a fragment of wire surface not covered by oxide layer, 2 - area filled with a large amount of oxides.

of oxides (2). As far as these areas are concerned, only a slightly smaller thickness of the layer might be observed. In the analyzed cases, the layer thickness in these areas was $23 \%$ to $25 \%$ smaller than an average thickness of the layer measured on the non-defected surfaces and the layers not covered with oxide.

\section{Conclusions}

1. The examination of wire surface revealed the presence of numerous defects, which 
occurred in the process of layer forming. The defects are in the form of intergranular gaps, partly or fully filled with oxides as a result of intergranular corrosion. The presence of laps, which are susceptible to more rapid oxidation of the material, has also been evidenced. In these areas, pits have been observed, which were formed as a result of erosive-corrosive interaction of molten polystyrene as well as tension during EPS block cutting process. Moreover, it has been shown that the surface of the wire is covered by oxide layer of nanometric thickness.

2. It has been shown that sputtering of Si target is possible by DC magnetron under a stable power and with supporting argon ionization by an additional source, such as another magnetron operating at reduced performance parameters.

3. The analysis of Si layer sections indicated that it crystallizes in the form of elongated compact grains in the configuration of inverted cone relative to the base. Within layers deposited on steel base, there are sublayers with finer grains, on which larger grains are built perpendicularly to the base. The layers are compact and they form a continuous coating that masks surface defects of the wire, even in the areas covered by oxide coating.

4. Taking into account the compact structure of Si layer as well as its continuity in the areas with numerous wire surface defects and oxide clusters, it can be assumed that it will contribute to the increase of wire resistance and corrosive-erosive factors related to molten polystyrene during cutting process.

\section{Acknowledgements}

This publication was written at the Technical University of Liberec, Faculty of Mechanical Engineering with the support of the Institutional Endowment for the Long Term Conceptual Development of Research Institutes, as provided by the Ministry of Education, Youth and Sports of the Czech Republic in the year of 2017. This publication was written as a part of the project "The study and evaluation of the material's structure and properties" with the support of the Specific University Research Grant, as provided by the Ministry of Education, Youth and Sports of the Czech Republic in the year of 2017. TEM examinations were carried out at the Institute of Metallurgy and Material Science of the Polish Academy of Science in Cracow.

\section{References}

[1] RESZKA K., The layer systems of aluminum oxide and platinum for catalytic applications, manufactured on $\mathrm{Fe}$ CrAl steel, Wydawnictwo Uczelniane Politechniki Koszalińskiej, Koszalin 2011.

[2] RESZKA K., Vacuum, 81 (2007), 1202.

[3] ResZKa K., MurzyŃśnI D., RAKOCZy J., Vacuum, 83 (2009), S224.

[4] Morgiel J., Major Ł., Grzonka J., Wojewoda J., POMORSKA M., Elektronika, 10 (2007), 46.

[5] ResZKa K., Adv. Sci. Technol., 45 (2006), 2110.

[6] SZCZYPIŃsKi M., RESZKA K., MURZYŃSKI D., SZCZYPIŃSKI M.M., Funct. Nanostruct., 2 (2017), 91.

Received 2017-03-18 Accepted 2018-03-15 